\title{
MINI-SYMPOSIUM
}

\section{Contrast echocardiography: from left ventricular opacification to myocardial perfusion. Are the promises to be realised?}

\section{J Monaghan}

Heart 2003;89:1389-1390

V rtually every cardiac imaging technique utilises contrast agents, yet the adoption of ultrasound contrast agents into the routine practice of echocardiography has been slow. The commercial and clinical promise of contrast echo has not yet been fulfilled and we are truly standing at the crossroad between success and failure of the technique. In this introductory article to the mini-symposium on contrast echocardiography, I attempt to give a general overview of the current clinical role of echo contrast agents. Three further articles examine in more critical detail where we currently stand with respect to the role of contrast for left ventricular opacification, for perfusion imaging during stress and in patients with acute myocardial infarction.

Patent issues, cost, reimbursement, difficult regulatory issues in the USA, and a smaller market than originally envisaged has led a number of contrast agent manufacturers to review their position. Some agents have been withdrawn from development and some put on hold. Instrument manufacturers have been reflecting upon their substantial investment in contrast imaging technology.

Fortunately, a number of key issues have occurred which indicates a far more optimistic future for contrast echo. These issues include agreement on reimbursement for the use of contrast in the USA, resolution of some patent disputes, new technology which makes myocardial perfusion imaging realistic, and more of a consensus on what is needed to make contrast echo successful.

In many countries there are currently three licensed contrast agents. Although all three have a licence for left ventricular opacification and Doppler enhancement, improvements in imaging technology, particularly second harmonics, have paradoxically reduced the need for contrast in routine studies.

Stress echocardiography remains an area when contrast can and should make a significant contribution. Stress echo is an operator dependent technique with poor image quality, especially at peak stress, being the limiting factor. Despite the

\section{Contrast echocardiography: the threats}

- Myocardial perfusion imaging is still a research rather than a clinical tool

- Cost

- Reimbursement

- Slow progress on licensing

- Patent issues

- Improvements in image quality: reduce need

- Intravenous line required

- Lack of education

\section{Contrast echocardiography: the opportunities}

- Enhance Doppler studies

- Tricuspid regurgitation, aortic stenosis, pulmonary vein flow

- Enhance endocardial definition

- Difficult patients

- Stress echo: major role

- Delineate anatomy

- Thrombus

- Fistulae, etc

- Myocardial perfusion

- Near future

- Drug delivery

- Far future

- Pressure measurement

- Far future

- Now reimbursed

- New agents becoming available

- Equipment progressing fast

clinical value of stress echo, its application is often limited by the lack of time and staff available to perform the studies plus the steep learning curve and the undoubted fact that the images can be difficult to interpret, especially at peak stress. Contrast can help overcome this and should become routine practice in stress echo examinations.

There are many studies showing a significant improvement in endocardial definition and interpreter confidence when contrast is used in stress studies. In our own department, contrast is used in at least $90 \%$ of stress patients. It adds little additional inconvenience to a pharmacological stress test since an intravenous line is already in place, and in terms of the real cost of performing a stress echo, the additional factor of contrast is not that significant. A stress echo study with contrast is still cheaper than a stress Sestamibi scan, and the techniques have similar sensitivity and specificity for detection of reversible ischaemia and myocardial viability. One

\section{Contrast agents licensed in the UK}

- Levovist (Schering): polysaccharide + air

- Optison (Amersham): albumin + octofluoropropane

- Sonovue (Bracco): lipid + sulfur hexafluoride 


\section{Contrast imaging methods}

- Harmonic power Doppler (angio): contrast destruction technique

- Grey scale harmonics (power pulse inversion, ultraharmonics): non-linear response technique

- Real time contrast imaging (power modulation, power pulse inversion, coherent imaging), low output power, non-destructive techniques

major indication for stress contrast studies in our own department is the evaluation of the physiological significance of known coronary lesions before proposed revascularisation. If the technique can help us make better management decisions over which patients would benefit from coronary bypass graft surgery (CABG) or coronary angioplasty (PTCA), then the additional cost of contrast is a small price to pay for increased diagnostic accuracy. This is especially true when you consider the cost of revascularisation. One saved PTCA or CABG would probably pay for a whole year's supply of ultrasound contrast media. The article by Harald Becher in this mini-symposium (see p 1394) critically examines the role of contrast for left ventricular opacification. He concludes that, particularly when used with new automated boundary detection techniques, we will soon reach the goal of automated and quantitative evaluation of regional and global left ventricular function.

The major market for contrast echo will occur when we can make myocardial perfusion imaging during rest or stress studies a reality. This has been described as the "holy grail" of contrast echo. The development cycle for contrast agents is much longer than that for ultrasound instrumentation. Therefore, most of the advances in this area have been in terms of increasing the sensitivity of the imaging technology to detect contrast microbubbles in the small quantities with which they occur in the myocardium. The contrast imaging technologies can be divided into three distinct groups, all of which rely upon the fact that contrast agents backscatter ultrasound in a different way to tissue. This issue is critical, since the technologies are trying to separate out noise from tissue from contrast microbubbles. However, the method of separating noise from tissue from contrast is different with each group of techniques. A more detailed description of the different imaging methods and how they may be combined with different stressors and different contrast administration methods is provided in my other article in this minisymposium (see p 1391).

In summary, we can use destructive high ultrasound power techniques which are very sensitive for detecting contrast within the myocardium but do not provide simultaneous wall motion information. Alternatively we can use low power realtime imaging, which is slightly less sensitive for myocardial contrast, but does provide wall motion information and excellent left ventricular opacification.

Real-time perfusion imaging has many potential advantages. The technique is relatively easy to use, many artefacts can be avoided, and wall motion information is obtainable alongside perfusion. The ability to supplement wall motion
Potential clinical roles for real-time perfusion

imaging

- Acute chest pain syndromes

- Viability assessment ?hibernation

- Endo/epi perfusion ratios

- Stress perfusion echo studies

- ?Quantification of myocardial blood flow

- Evaluation of success of thrombolysis

- Evaluation of success of PTCA/CABG

information with perfusion will make this technique particularly invaluable during stress echocardiography. In my opinion, stress myocardial contrast echocardiography has a number of important advantages over other functional tests. In the future, it will become the diagnostic test of choice for evaluation of reversible ischaemia and myocardial viability.

There are obviously many other chronic and acute clinical scenarios where real-time perfusion imaging, at the bedside, would have a tremendous impact upon patient management. One example is the use of contrast to detect myocardial viability post-myocardial infarction. This role is examined in this mini-symposium by Roxy Senior (see p 1398). He explains that if contrast is visible within the myocardium, this implies an intact microvasculature. The microvasculature will only remain intact if there are viable myocytes to supply. Several studies have recently demonstrated that the presence of myocardial contrast in akinetic segments can predict functional recovery and may also give useful information about the potential for re-modelling post-myocardial infarction. Clearly this technique would have several potential advantages over low dose dobutamine studies, including speed and the fact that it would be a truly bedside technique.

\section{CONCLUSION}

The clinical applications for contrast in echo are now well defined. The indications for contrast in routine Doppler enhancement, thrombus delineation, and left ventricular function studies are limited, given the quality of modern echocardiography systems. However, if you are performing stress echo, contrast is an invaluable aid and I believe that you will want to use it in the majority of studies. In the very near future, we will be using contrast routinely for myocardial perfusion studies, to enhance automated left ventricular function analysis systems and during real-time three dimensional echo studies. The far future holds the promise of drug delivery and intracardiac pressure measurement by ultrasound contrast agents.

There is no doubt that the role of contrast agents in echo imaging will be as important as contrast is in virtually every other medical imaging modality. The promises will be realised. The articles in this mini-symposium provides the critical analysis to back that statement up.

Correspondence to: Dr Mark J Monaghan, Cardiology Department, King's College Hospital, Denmark Hill, London SE5 9RS, UK; monaghan@compuserve.com 